\title{
Modeling of pulsar magnetospheres
}

\author{
Anatoly Spitkovsky \\ Princeton University, United States \\ email: anatoly@astro.princeton.edu
}

\begin{abstract}
The inner workings of pulsar magnetospheres have fascinated and confused researchers since the discovery of pulsars. I will review the status of magnetospheric models, including vacuum, space-charge-limited and resistive force-free MHD. I will highlight model predictions for the integrated pulsar quantities (such as spin down and torques) and the observational consequences of calculated magnetic field structure. Particularly, high-energy emission from pulsars allows putting new constraints on the geometry of the emission region and the physics of particle acceleration in the magnetosphere.
\end{abstract}

